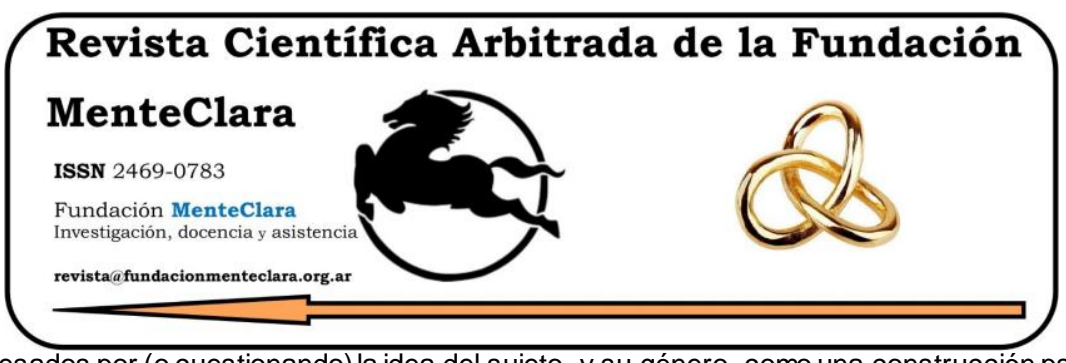

Artículos atravesados por (o cuestionando) la idea del sujeto -y su género- como una construcción psicobiológica de la cultura. Articles driven by (or questioning) the idea of the subject -and their gender- as a cultural psychobiological construction.

Vol. 6 (2021), enero-diciembre

ISSN 2469-0783

https: / / datahub.io/ dataset/2021-6-e234

\title{
LA IMPORTANCIA DE PROMOVER FELICIDAD Y SALUD EN EL ÁMBITO LABORAL
}

\author{
THE IMPORTANCE OF PROMOTING HAPPINESS AND HEALTH AT WORK
}

Melina S. Ignarski melinaignarski@gmail.com

Estudiante de Psicología, Cátedra Psicología de la Salud, Universidad de Flores, Argentina.

Cómo citar este artículo / Citation: Ignarski, M. (2021). La importancia de promover felicidad y salud en el ámbito laboral. Revista Científica Arbitrada de la Fundación

MenteClara, Vol. 6 (234). DOI: https://doi.org/10.32351/rca.v6.234

Copyright: (C) 2021 RCAFMC. Este artículo de acceso abierto es distribuido bajo los términos de la licencia Creative Commons Attribution 4.0 International License (CC BY 4.0). Recibido: 10/07/2021. Aceptado: 16/07/2021 Publicación online: 21/07/2021

Conflicto de intereses: Ninguno que declarar.

\section{Resumen}

Desde sus inicios la Psicología Positiva consideró como uno de sus problemas fundamentales, no solo a los aspectos negativos, sino a todo lo que hace que la vida valga la pena y tenga sentido. De este modo, se puede decir que dicha rama de la Psicología estudia tanto las dificultades como las fortalezas de las personas. Gracias a esta forma distinta de estudiar las experiencias humanas, es que se generan importantes aportes a la promoción de la salud, la cual apunta a la salud y a su salutogénesis del ser humano. Además, no busca evitar solo la enfermedad, sino también incrementar la salud y la felicidad, que es clave en la calidad de vida de los individuos, tanto en lo personal como en lo organizacional. El objetivo principal de este trabajo es exponer la relación existente entre la promoción de salud y la felicidad, y las distintas estrategias que pueden llevarse a cabo dentro del ámbito laboral, debido a que diversos estudios demuestran que la satisfacción laboral se corresponde 
con la felicidad y la dimensión emocional de la salud. Dicha relación entre la promoción de salud y la felicidad, forman parte del objetivo de la Psicología Positiva; la cual contribuye a mejorar la calidad de vida, su bienestar subjetivo, en conclusión: la salud del individuo.

\begin{abstract}
Since its beginnings, Positive Psychology has considered as one of its fundamental problems, not only the negative aspects, but everything that makes life worthwhile and meaningful. Thus, it can be said that this branch of psychology studies both the difficulties and the strengths of people. Thanks to this different way of studying human experiences, it generates important contributions to health promotion, which aims at the health and salutogenesis of the human being. In addition, it does not only seek to prevent disease, but also to increase health and happiness, which is key to the quality of life of individuals, both personally and organizationally. The main objective of this paper is to expose the relationship between health promotion and happiness, and the different strategies that can be carried out within the workplace, because several studies show that job satisfaction corresponds to happiness and the emotional dimension of health. This relationship between health promotion and happiness is part of the objective of Positive Psychology, which contributes to improve the quality of life, subjective well-being, in conclusion: the health of the individual.
\end{abstract}

Palabras Claves: Promoción de la salud; Felicidad; Bienestar subjetivo; Psicología Positiva; Ámbito laboral

Keyw ords: Health promotion; Happiness; Subjective well-being; Positive psychology; Work environment 


\section{Introducción}

La Psicología Positiva, planteada por Seligman en 1998, es un nuevo paradigma que la psicología propuso para estudiar las experiencias humanas. Procura no solo ocuparse de componer las peores cosas de la vida en los individuos, sino además en potenciar las cualidades positivas de dichas personas (Domínguez \& Ibarra, 2017). Estos autores además afirman que Martin Seligman es el principal representante de este nuevo paradigma, el cual pretende potenciar y promover las fortalezas humanas. A su vez sostienen que cuando hablamos de bienestar subjetivo, calidad de vida, satisfacción con la viday felicidad tenemos que referirnos a conceptos que son utilizados para definir lo que las personas valoran y aprecian de la vida.

Este nuevo enfoque, ha realizado desde hace ya 35 años, investigaciones acerca del bienestar psicológico. Una pregunta clave que se expone en el análisis de la felicidad es ¿Qué puede hacer una persona para que su vida sea plena? (Domínguez \& Ibarra, 2017). Además, mencionan que Martin Seligman, expone ciertos recursos para que esto suceda: vivir de forma placentera, comprometerse a gozar lo que se hace, reconocer las fortalezas de los individuos y poner en marcha las fortalezas personales para contribuir a los otros.

Asimismo, (Domínguez \& Ibarra, 2017) sostienen que recientemente Seligman añadió un componente más referente con los vínculos positivos: la socialización. Por otro lado, además aseguran que un supuesto primordial de la Psicología Positiva es que el bienestar humano y el estudio de grandiosidad son tan genuinos como la patología y los problemas psicológicos. 
Este trabajo se propuso como objetivo demostrar la importancia y las distintas estrategias que pueden llevarse a cabo en el ámbito laboral, basándose en las variables de felicidad y promoción de la salud, abordadas a partir del surgimiento de la psicología positiva.

\section{Felicidad}

En el ámbito científico, el término "felicidad" fue interpretado por bienestar subjetivo (Scorsolini-Comin \& dos Santos, 2010). Morán et al. (2017) afirman que este concepto es determinado como la vida exitosa y afecto positivo, sin afectos negativos. Es por ello que:

Los hallazgos empíricos indican que los niveles altos de felicidad se asocian, entre otras, a ventajas para la salud mental y con disposiciones y rasgos de personalidad más adaptativos. Concretamente, la satisfacción con la vida es el principal indicador del bienestar subjetivo. (Morán, Finez, \& Fernández-Abascal, 2017, pág. 59)

Por su parte, Díaz y Carrasco (2018) afirman que la felicidad es indispensable si tiene relación con la calidad de vida de un sujeto, tanto en lo personal como en lo organizacional, lo cual otorga superiores colaboraciones entre individuos e incrementa la constancia en la ejecución de fines, rendimiento, originalidad, innovación, disfruto gremial y motivación. Estos autores, también sostienen que: “...en los últimos años la Psicología Positiva organizacional ha desarrollado nuevas variables que afectan el desarrollo de las personas dentro de la organización siendo la felicidad una de estas nuevas dimensiones de análisis" (Díaz \& Carrasco, 2018, pág. 2). 
Desde hace unos años ha vuelto a nacer un interés por el estudio de la felicidad en el ámbito laboral. Gabini (2018) por su parte, consolida que la felicidad en el trabajo tiende a ocasionar un mayor rendimiento, autocontrol, buenas relaciones interpersonales, salud psicológica y física, y mayor persistenciay vitalidad. Asimismo, asegura que los individuos que poseen pensamientos positivos tienden a incidir en situaciones o ambientes novedosos. De modo que, son individuos exitosos en su ambiente laboral y más felices.

Gabini (2018), además afirma que la felicidad les posibilita a los trabajadores ver al mundo más seguro, poder tomar elecciones libremente y poder gozar de una vida más sana. No obstante, postula también que aquellas personas que no se declaran felices con sus empleos consideran al dinero como un factor que conduce a la felicidad.

En lo que respecta a los antecedentes, muchos investigadores dedicaron su tiempo y trabajo para desarrollar la teoría del bienestar. Dentro de los autores más destacados se encuentra Seligman, el cual presenta un modelo que generaría felicidad, al que llamó PERMA: pleasure -placer-, engagement -compromiso-, o Flow, relationships relaciones sociales-, y accomplishments -alcanzar metas-. Cada uno de estos elementos contribuye a lograr bienestar personal (Domínguez \& Ibarra, 2017).

Otra teoría es la denominada auto-determinación, por DeHaan y Ryan (Gabini, 2018) que indica que la posibilidad de aumentar la felicidad es más probable si se produce debido a cambios vitales eudaimónicos más que hedónicos.

Por su parte, Csikszentmihalyi investigó acerca de las experiencias óptimas o de Flow, que las define como experiencias donde un individuo 
dirige toda su atención en lo que está haciendo, se aísla de todo lo que lo rodea y disfruta de esa vivencia (Díaz \& Carrasco, 2018).

\section{Promoción de la Salud}

Como se señaló anteriormente, la felicidad es una de las claves en la calidad de vida de los individuos (Díaz \& Carrasco, 2018), conjuntamente con la promoción de la salud. Por lo cual es importante destacar como se fueron construyendo las bases de la misma.

En 1938 Sigerist, eminente médico e historiador suizo, inició la construcción teórico-práctica de la promoción de la salud cuya meta era lograr el bienestar de las personas (Coronel, 2017).

La Organización Mundial de la Salud (OMS) en 1948, definió a la salud como condición de bienestar físico, mental y social completo, y no solamente ausencia de la enfermedad (Calpa, y otros, 2019). Luego, en sentido cronológico se reproducen otras definiciones propuestas por la OMS. La declaración de Alma-Ata de 1978 reafirma la definición anterior de salud y agrega que es fundamental derecho humano y que la consecución del más alto nivel posible de salud es el más importante objetivo social del mundo (Coronel, 2017).

Por último, la Carta de Ottawa de 1986, define:

La promoción de la salud consiste en proporcionar a los pueblos los medios necesarios para mejorar su salud y ejercer un mayor control sobre la misma. Para alcanzar un estado adecuado de bienestar físico, mental y social un individuo o grupo debe ser capaz de identificar $y$ realizar sus aspiraciones, de satisfacer sus necesidades y de cambiar o adaptarse al medio ambiente. La salud se percibe pues, no como el 
objetivo, sino como la fuente de riqueza de la vida cotidiana. Se trata por tanto de un concepto positivo que acentúa los recursos sociales $y$ personales así como las aptitudes físicas. (Organización Mundial de la Salud, 1986)

Luego de esta definición, la OMS introduce la práctica de promoción de salud permitiendo al individuo controlar en primera persona la "propia salud", mejorándola. Después de varias definiciones de promoción de salud se coincidió de manera general que les permite a los individuos aumentar el control sobre su salud para poder mejorarla (Coronel, 2017).

Con respecto al ámbito laboral, al que apunta esta investigación, Calpa et al. (2019), afirman que la promoción de estrategias de estilos de vidas saludables (EVS), mejora la calidad en el ámbito laboral, las consecuencias relacionadas con la salud, aumenta el rendimiento de los empleados y alivia los costos de asistencia médica. Debido a que la mayoria de la gente pasa la mayor parte de su historia en el trabajo, el entorno laboral se convierte en un lugar en donde se comparten y se proporcionan diferentes estilos de vida. Para que esto suceda es necesario producir políticas para regular la salud de los empleados, fomentar y mantener la salud en el entorno de trabajo (Calpa, y otros, 2019).

Un ejemplo favorable, podria ser ocupar tácticas enfocándose en un proceso de acción de la salud, según Calpa et al. (2019) un programa de promoción de actividad física en el entorno laboral con población de mujeres, por ejemplo, el cual ha sido llevado a cabo y ha resultado positivo, en tanto mejoró sus funcionalidades cognitivas, estados de alerta y productividad laboral.

Por ende, este análisis nos posibilita pensar sobre la exigencia de realizar tácticas orientadas al control del estrés como elemento 
importante para la salud y el bienestar de los trabajadores debido a que, esto les genera un confort personal, y además conduce a la producción de las empresas (Calpa, y otros, 2019).

En el ámbito laboral, Benavides et al. (2017) determinan que las actividades de promoción de salud tienen como finalidad desplegar medidas a nivel orgánico e individual para capacitar a aquellas personas encargadas de trabajar en el cuidado de su salud. Asimismo, mencionan a la salud laboral; la cual se encarga de prever enfermedades, lesiones en los trabajadores y promover la salud de cada uno de ellos. Por ende, se puede afirmar que su objetivo es ayudar a que las personas puedan disfrutar de una vida laboral prolongada y saludable.

\section{Relación entre felicidad y promoción de la salud}

La aparición de la Psicología Positiva, propuso un cambio de enfoque, especialmente en la década de los años 90 corrigiendo los aspectos negativos y enfermedades para los aspectos positivos y la promoción de la salud de las personas (Scorsolini-Comin \& dos Santos, 2010). Estos autores afirman que el constructo bienestar subjetivo fue pensado como el estudio científico de la felicidad, relacionado firmemente a la promoción de la salud, tratándose de una experiencia interna de cada persona que juzga como se siente el individuo y del grado de satisfacción con su vida.

Scorsolini-Comin y dos Santos sostienen que los aportes de producción científica se preocupan por la forma en que el concepto de felicidad tiene o debe estar aplicado en la promoción de la salud, con la atención encabezada en determinar en qué medida una técnica puede mejorar el bienestar subjetivo de los individuos, lo que afecta 
directamente en la calidad de vida y salud (Scorsolini-Comin \& dos Santos, 2010).

\section{Conclusión}

En el ámbito laboral se deben generar más estudios que conduzcan al conocimiento sobre la promoción de estilos de vida saludables, desplegar estrategias para mejorar la calidad de vida humana y experimentar acciones que impliquen a todos los niveles jerárquicos de la organización, desde una mirada colectiva de entrono de trabajo saludable (Calpa, y otros, 2019)

Para lograr una promoción de salud en este ámbito, es importante que la salud laboral, de forma integral, aborde la salud de los empleados ya que, es necesario prevenir los riesgos laborales y extralaborales y asistir los problemas de salud que estén relacionados o no con el trabajo (Benavidez, Delclós, J., \& Serra, C., 2017).

Con lo que respecta a la felicidad en el ámbito laboral, su concepto abarca varios fenómenos; desde emociones transitorias hasta más formales. En este ámbito, el bienestar subjetivo suele estar dominado por eventos puntuales o crónicos que hacen a la actividad, empleo y a la estructura (Gabini, 2018).

Estudios demuestran que la satisfacción laboral está correspondida con la felicidad y la dimensión emocional de la salud, por su parte, en el trabajo incrementa una alta satisfacción en las probabilidades de reportar niveles buenos de energía, amplia la calidad y cantidad de interacciones sociales y concede a los trabajadores un amparo adicional contra la ansiedad, la depresión y la pérdida de control emocional y 
conductual (Díaz \& Carrasco, 2018). Estos autores consideran que lo más importante es fomentar la felicidad en los trabajadores, ya que las emociones positivas actúan como un antídoto frente a las emociones negativas. Además, señalan que la felicidad en el ámbito laboral puede relacionarse con un alto grado de creatividad y acrecentamiento debido a un buen comportamiento y proceder con compañeros de trabajo y clientes.

En conclusión, un individuo con un elevado nivel de bienestar psicológico y subjetivo, saludable y con un control interno y externo podría sentirse adecuadamente consigo mismo, hallar un motivo para vivir, relacionarse de manera positiva con las personas y desarrollar autonomía entre otros aspectos. 


\section{Referencias}

Benavides, F., Delclós, J., \& Serra, C. (2017). Estado de bienestar y salud pública: el papel de la salud laboral. Gaceta Sanitaria, 32(4), 337-380. doi: https://dx.doi.org/10.1016/j.gace ta.2017.07.007

Calpa, A., Santacruz, G., Álvarez, M., Zambrano, C., E, H., E., H., \& S., M. (2019). Promoción de estilos de vida saludables: estrategias y escenarios. Hacia la promoción de la salud, 24(2), 139-155. doi:https://doi.org/10.17151/hpsal.20 19.24.2.11

Corone1, J. M. (2017). La promoción de la salud: evolución y retos en América Latina. MEDISAN, 21(7), 926-932. Obtenido de http://scielo.sld.cu/scielo.php?script=sci_arttext\&pid=S102930192017000700018\&lng=es $\&$ tlng=es

Díaz, F., \& Carrasco, M. (2018). Efectos del clima organizacional y los riesgos psicosociales sobre la felicidad en el trabajo. Contaduría y administración, 63(4), 1-14. doi: https://doi.org/10.22201/fca.24488410e.2018.1142

Domínguez, R., \& Ibarra, E. (2017). La psicología positiva: Un nuevo enfoque para el estudio de la felicidad. Razón y palabra, 21(96), 660-679. doi:

https://journals.copmadrid.org/clysa/art/j.clysa.2016.11.003

Gabini, S. (2018). Felicidad en el trabajo: breve actualización desde la psicología positiva. Revista de Psicología, 14(27), 69-75. Obtenido de https://repositorio.uca.edu.ar/bitstream/123456789/6084/1/felicidad-trabajo-psicologiapositiva.pdf

Morán, M., Finez, M., \& Fernández-Abascal, E. (2017). Sobre la felicidad y su relación con tipos y rasgosde personalidad. Clínica y salud, 28(2), 59-63. doi:

https://doi.org/10.1016/j.clysa.2016.11.003

Organización Mundial de la Salud. (1986). Carta de Ottawa para el Fomento de la salud. Primera Conferencia Internacional sobre Fomento de la Salud. Ottawa.

Scorsolini-Comin, F., \& dos Santos, M. (2010). El estudio científico de la felicidad y la promoción de la salud: revisión integradora de la literatura. Rev. Latino-Am. Enfermagem, 18(3), 192-199. Obtenido de https://www.scielo.br/j/rlae/a/TvRZgKWyptwx6YMYsLMkRZG/?lang=es\&format=pdf 